\title{
Variación intraespecífica en Cryptotis aroensis (Mammalia, Soricomorpha, Soricidae) de Venezuela
}

Franger J. García ${ }^{*}$, Mariana Isabel Delgado-Jaramillo ${ }^{1,2}$ y Marjorie Machado

Introduction: In Venezuela, the genus Cryptotis is represented by four species: C. meridensis, C. tamensis, C. aroensis and C. venezuelensis. C. meridensis, and C. tamensis have been documented in the Andes (Cordillera de Mérida, El Tamá, and Sierra de Perijá). On the other hand, C. aroensis, and C. venezuelensis are recorded only in the Cordillera de la Costa (Sierra de Aroa, and Serranía del Litoral). Cryptotis aroensis, was recently described and is known from only three specimens from type locality (Sector Milla, Sierra de Aroa, Yaracuy State), in Northern Venezuela. Based on a new series (45 specimens) from Yurubí National Park, Sierra de Aroa, Yaracuy State, Venezuela, in this article, we evaluated the intraspecific variations of Cryptotis aroensis, previously unknown in the literature.

Metodology: Specimens studied are preserved in the Museo de la Estación Biológica de Rancho Grande (EBRG, Aragua State, Venezuela), and the Museo de Zoología, Universidad de Carabobo (MZUC, Carabobo State, Venezuela). External measurements $(\mathrm{mm})$ and weight $(\mathrm{g})$ were taken from the colector's tag. Craniodental terminology follows Woodman (2002, 2003), Quiroga-Carmona and Molinari (2012), and Woodman et al. (2013), and the craniodental measurements were taken with a digital caliper. Finally, for each measure, mean and range were calculated and we used a Student $t$ test with intervals confidence not less than $95 \%$ to explore differences related to sex.

Results: Our morphological and metric analysis indicates that specimens of Cryptotis aroensis from Yurubí National Park are smaller and with longer tails, compared with C. meridensis, C. tamensis and C. venezuelensis. There were no significant differences between sexes for the individuals studied, suggesting that there is no sexual dimorphism in C. aroensis. Within this sample there were highly variable morphological characters such as the shape of the wart on the nasolabial groove in the rhinarium, numbers of postnasal warts on the muzzle, hindlimb digits, presence and position of the fourth unicuspid tooth, presence and size of dorsal foramina on the skull, presence and size of foramina on posterior border of tympanic process of petromastoids. The humerus was slightly smaller, less robust and slightly curved in C. aroensis compared with one humerus available for C. meridensis.

Discussion: The high intraspecific variation in C. aroensis is consistent with previous reports that indicates a high morphological and metric variation among individuals in the groups Cryptotis nigrescens, C. goldmani, C. thomasi, C. mexicana, and C. parva. With respect to the characters in the original description of $C$. aroensis, there are similarities when the sample increases from three (holotype and two paratypes) to 45 specimens. Such characters include body-head length, and length of mandible, besides the presence of cusps on the third upper molar that define a complex molar. Additionally, the result of this study suggests that tail length (extremely long) can be useful to separate $C$. aroensis of the different taxa in Venezuela. When we compared the type series of $C$. venezuelensis, a species known only from three specimens from the Cordillera de la Costa, like C. aroensis, there are several similarities in morphology and metrics. Those characters include its size and body coloration, presence and visibility of the fourth unicuspid tooth, shape

'Laboratorio Museo de Zoología, Departamento de Biología, Facultad Experimental de Ciencias y Tecnología (FACyT). Universidad de Carabobo, Campus Bárbula, municipio Naguanagua, Venezuela, Valencia E-mail: cormura@yahoo.com (FJG), marjoriesilvera@gmail.com (MM).

2Postgrado en Biología Animal (PPGBA), Departamento de Zoología, Universidad Federal de Pernambuco (UFPE), Pernambuco, Brasil. E-mail: marianadelgado13@yahoo.es (MID).

${ }^{*}$ Corresponding autor 
of the wart and its extension on the nasolabial groove in the rhinarium, the numbers of postnasal warts, size and shape of the angular and coronoid processes, and the average of scales per centimeter on the tail. These characters suggest that $C$. aroensis and $C$. venezuelensis might be closely related taxa.

Key words: Cryptotis aroensis, morphological variation, Sierra de Aroa, Yaracuy, Yurubí National Park.

Resumen

Se evalúan las variaciones intraespecíficas de Cryptotis aroensis, especie descrita sobre la base de tres individuos en Venezuela. El estudio se realizó con 45 especímenes del Parque Nacional Yurubí, Estado Yaracuy, lo que permitió aportar información sobre variación morfológica y métrica. Se compararon estos resultados con la descripción original de éste y las especies geográficamente cercanas en Venezuela (C. venezuelensis, C. meridensis y C. tamensis). Se reportan rasgos altamente variables no documentados previamente que ayudan a enriquecer el conocimiento taxonómico de esta especie. Se encontró que un conjunto de caracteres morfológicos, como la forma de la verruga y extensión en el surco nasolabial del rinario, número de verrugas post-nasales, presencia del cuarto diente unicúspide y el tercer molar superior complejo, forma de los procesos angulares y coronoides entre otros, son similares entre $C$. aroensis y $C$. venezuelensis. Estos caracteres podrían sugerir que ambos taxones están cercanamente relacionados.

Palabras clave: Cryptotis aroensis, Parque Nacional Yurubí, Sierra de Aroa, variación morfológica, Yaracuy.

Introducción

Las musarañas de orejas cortas del género Cryptotis (Orden Soricomorpha), han sido separadas en el Neotropico en cuatro grupos o complejos informales (Woodman y Timm 1999; Woodman 2002, 2003; Quiroga-Carmona y Molinari 2012, Quiroga-Carmona 2013). Los grupos presentes en Sur América pertenecen a dos complejos: Cryptotis nigrescens, que incluye a C. nigrescens, C. mayensis, C. orophilus, C. magnus, C. merriami, C. hondurensis, C. merus, C. colombianus y C. brachyonyx, y C. thomasi, que agrupa a C. thomasi, C. medellinius, C. meridensis, C. tamensis, C. peruviensis, C. montivagus, C. squamipes, C. equatoris, C. venezuelensis y C. aroensis.

En Venezuela se reconocen cuatro especies, conformadas por C. meridensis Thomas 1898, C. tamensis Woodman 2002, C. aroensis Quiroga-Carmona y Molinari 2012 y C. venezuelensis Quiroga-Carmona 2013 (Sánchez-Hernández y Lew 2012; QuirogaCarmona y Molinari 2012; Quiroga-Carmona 2013). C. meridensis, C. aroensis y C. venezuelensis son especies endémicas. La primera es conocida para la Cordillera de Mérida, en los Estados Trujillo, Mérida y Táchira (Woodman 2002), la segunda de la Sierra de Aroa en el Estado Yaracuy (Quiroga-Carmona y Molinari 2012) y la tercera para la Serranía del Litoral en el Estado Aragua y Distrito Capital (Quiroga-Carmona 2013).

Por su parte, C. tamensis habita en el Páramo de El Tamá (oeste del Estado Táchira, en el Macizo de El Tamá), extendiendo su distribución al norte de la Cordillera Oriental de Colombia (Woodman 2002).

Cryptotis aroensis se describió recientemente con base en una pequeña serie de tres individuos (holotipo y dos paratipos), provenientes de un bosque nublado de la Sierra de Aroa, Estado Yaracuy en el noroccidente de Venezuela (Quiroga-Carmona y Molinari 
2012). Hasta la fecha la información ecológica y taxonómica sobre este taxón es muy incipiente y no existen estudios que documenten la variación morfológica que pueda exhibir C. aroensis en su intervalo de distribución. Este último punto se hace importante ya que para el género en Venezuela se han documentado altas variaciones en algunos atributos utilizados como caracteres diagnósticos (Woodman 2002).

En el marco de un proyecto sobre el estudio de los pequeños mamíferos del extremo occidental de la Cordillera de la Costa de Venezuela, realizado en el Parque Nacional Yurubí, entre los años 2011-2012 (García et al. 2013), fueron colectados 45 individuos adultos referibles a $C$. aroensis. La adición de esta muestra permitió revisar algunos aspectos de la variabilidad intraespecífica no documentados previamente para la especie (Quiroga-Carmona y Molinari 2012) y de esta manera ampliar el conocimiento taxonómico y de distribución de C. aroensis en la Sierra de Aroa.

Figura 1. Ubicación geográfica de la nueva población de $C$. aroensis (círculo) en el Parque Nacional Yurubí, Sierra de Aroa, Venezuela. La estrella indica la localidad tipo (Capilla de Milla, Sector Milla, Cocorote). Las áreas grises corresponden con alturas mayores a $500 \mathrm{~m}$ s.n.m.

Los especímenes estudiados provienen de un bosque nublado en un intervalo de altitud de 1400-1940 m, en el Parque Nacional Yurubí, Sierra de Aroa, Estado Yaracuy (La Trampa del Tigre, Sector El Silencio, $10^{\circ} 24^{\prime} 11^{\prime \prime} \mathrm{N}-68^{\circ} 48^{\prime}$ 01" O; Fig. 1). Los ejemplares se encuentran depositados en el Museo de la Estación Biológica de Rancho Grande (EBRG, Maracay, Venezuela) y Museo de Zoología de la Universidad de Carabobo (MZUC, Valencia, Venezuela) (Apéndice).

Las medidas externas y cráneo-mandibulares seleccionadas para esta nueva serie de C. aroensis y la nomenclatura de las partes óseas y dentarias siguen a las descripciones aportadas por Woodman (2002, 2003), Woodman et al. (2003) y Quiroga-Carmona y

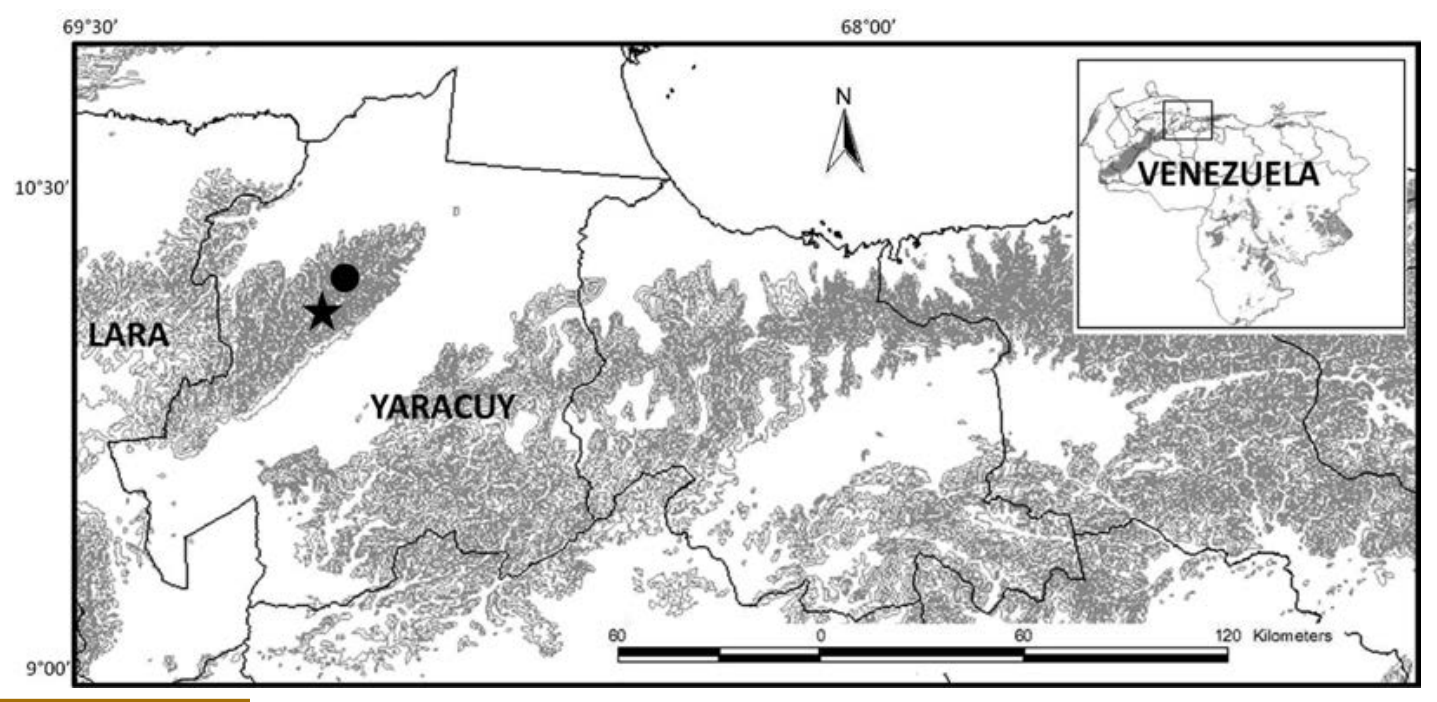

Molinari (2012). Estas medidas fueron tomadas con un calibrador digital de $0.01 \mathrm{~mm}$ de precisión, bajo un microscopio estereoscópico (LABOMED CZM6 4.5X). Las mismas fueron: longitud cabeza-cuerpo, longitud de la cola, longitud de la pata posterior, longitud de la oreja, longitud condilobasal, ancho de la caja cefálica, ancho del plato cigomático, ancho interorbitario, ancho del paladar en los primeros dientes unicúspides, ancho del paladar en los segundos molares superiores, longitud del paladar, longitud de la fila de dientes maxilar, longitud de la fila de dientes unicúspide, longitud de la fila de molares superiores, longitud de la mandíbula, altura del proceso coronoide, altura 
del valle coronoide, altura del cóndilo articular, distancia del cóndilo articular al borde posterior del tercer molar inferior, longitud de la fila de dientes en la mandíbula, longitud del primer molar inferior y peso en gramos.

Para cada medida se calculó la media y el intervalo de variación y se realizó una prueba de $t$ de Student con intervalos de confianza no menores a $95 \%$ para explorar diferencias relacionadas con el sexo y de esta manera tener un criterio para separar o no a las muestras. La información generada en este trabajo permitió hacer comparaciones sobre variación con las otras especies del grupo $C$. thomasi presentes en Venezuela $(C$. meridensis, $C$. tamensis y $C$. venezuelensis). Para esto último, se revisaron especímenes depositados en MZUC, particularmente C. meridensis (Apéndice) y para el resto de los taxones, se consultó literatura previa y posterior a la descripción de $C$. aroensis (Woodman 2002; Quiroga-Carmona 2013).

Resultados

En la Tabla 1 se presentan las medidas externas, craneanas y mandibulares para los ejemplares estudiados de $C$. aroensis (incluyendo la serie tipo), así como para las otras especies descritas para Venezuela referidas en la literatura. La prueba de $t$ de Student no arrojó diferencias significativas entre las medidas de ambos sexos $(P>0.05)$ y por lo tanto, los resultados son tratados de aquí en adelante como un único conjunto.

Los ejemplares de $C$. aroensis son de tamaño pequeño en las longitudes cabezacuerpo y peso, cuando se comparan con las especies andinas C. meridensis y C. tamensis; y similar a $C$. venezuelensis (Tabla 1 ). La verruga sobre el surco nasolabial presenta las siguientes variaciones: larga y delgada, extendida desde la base del rinario hasta la boca (EBRG-29385, 29389, 29396; MZUC-1010, 1011, 1018, 1021, 1024, 1027); o larga, pero no alcanzando la boca (EBRG-29384, 29390, 29393, 29394; MZUC-1013, 1015, $1016,1019,1022,1023,1025,1026,1028)$; o pequeña, de forma ovalada y restringida a la base del rinario (EBRG-29383, 29386, 29387, 29388, 29391, 29392, 29395, 29397; MZUC-1012, 1014, 1017, 1020, 1029, 1031, 1038; Fig. 2).

El hocico posee variaciones en las verrugas post-nasales: con cuatro pares en ambos lados (EBRG-29394); o cinco pares (EBRG-29383; MZUC-1022, 1031); o seis pares (EBRG-29389, 29391, 29393, 29395; MZUC-1010, 1012, 1018, 1020, 1021, 1023, 1024, 1025, 1028, 1029); o siete pares (EBRG-29384, 29385, 29386, 29390; MZUC1011, 1013, 1014,1016, 1017, 1019, 1026, 1030); u ocho pares (EBRG-29388, 29393, 29396, 29397; MZUC-1015) o diez pares (EBRG-29387).

La longitud de las manos, incluyendo las garras, varió entre 8.0 y $11.0 \mathrm{~mm}$ y fueron consideradas pequeñas cuando se comparan con ejemplares disponibles de $C$. meridensis (Fig. 3A). Todos los individuos de C. aroensis, presentaron un par de vibrisas sobre el lado ventral de la muñeca; tienen las escamas de las manos pequeñas, de forma redonda, abarcando una superficie amplia de las manos. Las escamas de los dedos de las manos en vista ventral son cuadradas y se disponen en dos hileras. Las medidas disponibles de un individuo (MZUC-1038), en esta nueva serie para el dígito III de la mano fueron: metacarpal $=3.93 \mathrm{~mm}$; falange proximal $=2.23 \mathrm{~mm}$; falange media $=$ $1.29 \mathrm{~mm}$ y falange distal $=1.20 \mathrm{~mm}$ (longitud total del dígito $=8.65 \mathrm{~mm}$ ).

Las longitudes de los pelos dorsales variaron de 3-6 mm y los ventrales de 3-4 mm. En todos los individuos la coloración dorsal fue gris oscura (negruzca), con un lustre 
marrón claro, observado cuando el dorso es visto a contra luz. El vientre es de color gris, ligeramente más pálido que el dorso. Los pelos dorsales y ventrales presentaron dos

Tabla 1. Medidas externas, craneanas y mandibulares para Cryptotis aroensis y el resto de las especies descritas para Venezuela. Para todas las medidas se indica la media $y$ los valores inferiores y superiores. Todas las medidas están expresadas en milímetros (mm).

\begin{tabular}{|c|c|c|c|c|}
\hline Medidas & $\begin{array}{c}\text { C. aroensis } \\
\text { (EBRG, MZUC, } \\
\text { Quiroga-Carmona y } \\
\text { Molinari 2012) }\end{array}$ & $\begin{array}{c}\text { C. venezuelensis } \\
\text { (Quiroga-Carmona } \\
\text { 2013) }\end{array}$ & $\begin{array}{c}\text { C. meridensis } \\
\text { (Woodman 2002) }\end{array}$ & $\begin{array}{c}\text { C. tamensis } \\
\text { (Woodman 2002) }\end{array}$ \\
\hline Externas & $\mathrm{n}=41$ & $\mathrm{n}=2$ & $\mathrm{n}=65$ & $\mathrm{n}=21$ \\
\hline $\begin{array}{l}\text { Longitud cabeza- } \\
\text { cuerpo }\end{array}$ & $\begin{array}{c}73.00 \\
62.00-81.00\end{array}$ & $\begin{array}{c}78.00 \\
72.00-84.00\end{array}$ & $\begin{array}{c}89.00 \\
76.00-102.00\end{array}$ & $\begin{array}{c}86.00 \\
80.00-91.00\end{array}$ \\
\hline Longitud de la cola & $\begin{array}{c}42.29 \\
34.00-47.00\end{array}$ & $\begin{array}{c}33.50 \\
33.00-34.00\end{array}$ & $\begin{array}{c}34.00 \\
25.00-41.00\end{array}$ & $\begin{array}{c}36.00 \\
32.00-39.00\end{array}$ \\
\hline $\begin{array}{l}\text { Longitud de la pata } \\
\text { posterior }\end{array}$ & $\begin{array}{c}14.07 \\
13.00-16.00\end{array}$ & $\begin{array}{c}14.50 \\
14.00-14.50\end{array}$ & - & - \\
\hline Longitud de la oreja & $\begin{array}{c}7.06 \\
6.00-8.00\end{array}$ & $\begin{array}{c}6.75 \\
6.50-7.00\end{array}$ & - & - \\
\hline Peso (g) & $\begin{array}{c}11.14 \\
9.00-14.00\end{array}$ & $\begin{array}{c}10.00 \\
9.00-11.00\end{array}$ & $\begin{array}{c}12.30 \\
8.50-18.00 \\
\quad(n=48)\end{array}$ & $\begin{array}{c}13.90 \\
10.80-16.10 \\
(n=6)\end{array}$ \\
\hline Craneanas & $\mathrm{n}=24$ & $\mathrm{n}=3$ & $\mathrm{n}=35$ & $\mathrm{n}=13$ \\
\hline Longitud condilobasal & $\begin{array}{c}21.52 \\
20.90-21.99 \\
(n=23)\end{array}$ & $\begin{array}{c}21.23 \\
21.00-21.50\end{array}$ & $\begin{array}{c}21.50 \\
20.50-23.50\end{array}$ & $\begin{array}{c}21.50 \\
20.80-22.90\end{array}$ \\
\hline $\begin{array}{l}\text { Ancho de la caja } \\
\text { cefálica }\end{array}$ & $\begin{array}{c}10.29 \\
10.00-10.80 \\
(n=23)\end{array}$ & $\begin{array}{c}10.80 \\
10.70-10.90 \\
(n=2)\end{array}$ & $\begin{array}{c}10.40 \\
10.00-11.00\end{array}$ & $\begin{array}{c}10.50 \\
10.10-10.80 \\
(n=11)\end{array}$ \\
\hline $\begin{array}{l}\text { Ancho del plato } \\
\text { cigomático }\end{array}$ & $\begin{array}{c}2.18 \\
1.80-2.54\end{array}$ & $\begin{array}{c}2.23 \\
2.10-2.40\end{array}$ & $\begin{array}{c}2.00 \\
1.60-2.40\end{array}$ & $\begin{array}{c}2.10 \\
1.90-2.30\end{array}$ \\
\hline Ancho interorbitario & $\begin{array}{c}4.87 \\
4.60-5.10\end{array}$ & $\begin{array}{c}5.03 \\
5.00-5.10\end{array}$ & $\begin{array}{c}5.00 \\
4.70-5.40\end{array}$ & $\begin{array}{c}5.00 \\
4.80-5.30\end{array}$ \\
\hline $\begin{array}{l}\text { Ancho del paladar } \\
\text { en los primeros } \\
\text { unicúspides }\end{array}$ & $\begin{array}{c}2.56 \\
2.13-2.92\end{array}$ & $\begin{array}{c}2.85 \\
2.80-2.90 \\
(n=2)\end{array}$ & $\begin{array}{c}2.90 \\
2.60-3.10\end{array}$ & $\begin{array}{c}3.00 \\
2.70-3.10\end{array}$ \\
\hline $\begin{array}{l}\text { Ancho del paladar en } \\
\text { los segundos molares } \\
\text { superiores }\end{array}$ & $\begin{array}{c}6.05 \\
5.55-6.43\end{array}$ & $\begin{array}{c}6.36 \\
6.30-6.50\end{array}$ & $\begin{array}{c}6.40 \\
6.00-7.00\end{array}$ & $\begin{array}{c}6.40 \\
6.10-6.60\end{array}$ \\
\hline Longitud del paladar & $\begin{array}{c}9.22 \\
8.41-9.52\end{array}$ & $\begin{array}{c}9.73 \\
9.50-9.80\end{array}$ & $\begin{array}{c}9.50 \\
8.80-10.20\end{array}$ & $\begin{array}{c}9.50 \\
9.10-9.80\end{array}$ \\
\hline $\begin{array}{l}\text { Longitud de la fila de } \\
\text { dientes maxilar }\end{array}$ & $\begin{array}{c}8.21 \\
7.28-9.00\end{array}$ & $\begin{array}{c}8.50 \\
8.40-8.60 \\
(n=2)\end{array}$ & $\begin{array}{c}8.20 \\
7.30-8.80\end{array}$ & $\begin{array}{c}8.20 \\
7.90-8.70\end{array}$ \\
\hline $\begin{array}{l}\text { Longitud de la fila de } \\
\text { dientes unicúspide }\end{array}$ & $\begin{array}{c}2.70 \\
2.29-3.30\end{array}$ & $\begin{array}{c}2.50 \\
2.50-2.50 \\
\quad(n=2)\end{array}$ & $\begin{array}{c}2.80 \\
2.60-3.00\end{array}$ & $\begin{array}{c}2.80 \\
2.60-3.00\end{array}$ \\
\hline $\begin{array}{l}\text { Longitud de la fila de } \\
\text { molares superiores }\end{array}$ & $\begin{array}{c}5.78 \\
5.37-6.03\end{array}$ & $\begin{array}{c}5.95 \\
5.90-6.00 \\
(n=2)\end{array}$ & $\begin{array}{c}5.80 \\
5.50-6.30\end{array}$ & $\begin{array}{c}5.90 \\
5.60-6.10\end{array}$ \\
\hline
\end{tabular}




\begin{tabular}{|c|c|c|c|c|}
\hline Medidas & $\begin{array}{c}\text { C. aroensis } \\
\text { (EBRG, MZUC, } \\
\text { Quiroga-Carmona y } \\
\text { Molinari 2012) }\end{array}$ & $\begin{array}{c}\text { C. venezuelensis } \\
\text { (Quiroga-Carmona } \\
\text { 2013) }\end{array}$ & $\begin{array}{c}\text { C. meridensis } \\
\text { (Woodman 2002) }\end{array}$ & $\begin{array}{c}\text { C. tamensis } \\
\text { (Woodman 2002) }\end{array}$ \\
\hline $\begin{array}{l}\text { Longitud de la } \\
\text { mandíbula }\end{array}$ & $\begin{array}{c}7.36 \\
6.94-7.72\end{array}$ & $\begin{array}{c}7.80 \\
7.80-7.80 \\
(n=2)\end{array}$ & $\begin{array}{c}6.90 \\
6.30-7.60\end{array}$ & $\begin{array}{c}7.00 \\
6.90-7.40\end{array}$ \\
\hline $\begin{array}{l}\text { Altura del proceso } \\
\text { coronoide }\end{array}$ & $\begin{array}{c}4.57 \\
4.08-4.90\end{array}$ & $\begin{array}{c}4.65 \\
4.50-4.80 \\
(n=2)\end{array}$ & $\begin{array}{c}4.80 \\
4.50-5.20\end{array}$ & $\begin{array}{c}4.60 \\
4.30-4.80\end{array}$ \\
\hline $\begin{array}{l}\text { Altura del valle } \\
\text { coronoide }\end{array}$ & $\begin{array}{c}3.09 \\
2.90-3.30\end{array}$ & $\begin{array}{c}3.15 \\
3.10-3.20 \\
(n=2)\end{array}$ & $\begin{array}{c}3.30 \\
3.10-3.50\end{array}$ & $\begin{array}{c}3.10 \\
3.00-3.20\end{array}$ \\
\hline $\begin{array}{l}\text { Altura del cóndilo } \\
\text { articular }\end{array}$ & $\begin{array}{c}4.16 \\
3.90-4.54\end{array}$ & $\begin{array}{c}4.15 \\
3.90-4.40 \\
(n=2)\end{array}$ & $\begin{array}{c}4.40 \\
4.00-4.80\end{array}$ & $\begin{array}{c}4.40 \\
4.20-4.60\end{array}$ \\
\hline $\begin{array}{l}\text { Distancia del cóndilo } \\
\text { articular al borde } \\
\text { posterior del tercer } \\
\text { molar inferior }\end{array}$ & $\begin{array}{c}5.14 \\
4.55-5.60\end{array}$ & $\begin{array}{c}5.10 \\
5.10-5.10 \\
(n=2)\end{array}$ & $\begin{array}{c}5.60 \\
5.10-6.30\end{array}$ & $\begin{array}{c}5.40 \\
5.20-5.70\end{array}$ \\
\hline $\begin{array}{l}\text { Longitud de la fila } \\
\text { de dientes en la } \\
\text { mandíbula }\end{array}$ & $\begin{array}{c}6.43 \\
5.00-6.93\end{array}$ & $\begin{array}{c}6.65 \\
6.60-6.70 \\
(n=2)\end{array}$ & $\begin{array}{c}6.50 \\
6.10-6.90\end{array}$ & $\begin{array}{c}6.60 \\
6.30-6.90\end{array}$ \\
\hline $\begin{array}{l}\text { Longitud del primer } \\
\text { molar inferior }\end{array}$ & $\begin{array}{c}1.95 \\
1.71-2.22\end{array}$ & $\begin{array}{c}1.75 \\
1.70-1.80 \\
(n=2)\end{array}$ & $\begin{array}{c}2.00 \\
1.80-2.10\end{array}$ & $\begin{array}{c}1.90 \\
1.80-2.10\end{array}$ \\
\hline
\end{tabular}

bandas; una base gris amplia y la parte distal de color marrón. La coloración ventral es ligeramente más clara que el dorso.

Las escamas dorsales de los miembros posteriores son de forma redondeada y están acompañadas de un triplete de pelos en la base anterior de cada escama; en la última escama de la falange distal el número de pelos varía entre tres y siete. Los dígitos de los miembros posteriores en algunos individuos fueron delgados o robustos. Dentro de los dígitos delgados, se presentaron las siguientes variaciones en las escamas ventrales: redondas, juntas y arregladas en dos hileras (EBRG-29383, 29385); o cuadradas, juntas y arregladas en dos hileras (EBRG-29387, 29389; MZUC-1012, 1030, 1031); o cuadradas, muy juntas y arregladas en dos hileras (EBRG-29388); o cuadradas y ovaladas, juntas y arregladas en dos hileras (MZUC-1016, 1022). Las escamas en vista ventral de los dígitos robustos, presentaron las siguientes variaciones: redondas, separadas y arregladas en dos hileras (EBRG-29385, 29386, 29393, 29397); o redondas y cuadradas, separadas y arregladas en dos hileras (MZUC-1026, 1027); o sin un arreglo definido en los dígitos II, III, IV y V (EBRG-29384); u ovaladas, separadas y arregladas en dos hileras (EBRG-29396); o cuadradas, separadas y arregladas en dos hileras (MZUC-1013, 1024, 1025; EBRG29390, 29391, 29392); o cuadradas, ovaladas y triangulares, separadas y arregladas en dos hileras (MZUC-1019); o cuadradas y ovaladas, separadas y arregladas en dos hileras (MZUC-1021); o rectangulares y ovaladas, separadas y arregladas en dos hileras (MZUC-1010, 1011, 1014).

La cola es marcadamente larga (42.29 mm en promedio) y delgada cuando se compara con C. meridensis (Fig. 3B) y el resto de las especies de Venezuela (Tabla 1). La longitud 
de la cola promedió el $60 \%$ de la longitud cabeza-cuerpo; es densamente peluda desde la base hasta la punta, con escamas en la parte dorsal, que varían en forma (rectangular o redonda) y tamaño. Las escamas dorsales de la cola, tienen uno a tres pelos esparcidos sobre el borde anterior de ésta y tienen 30 escamas/centímetro en promedio. El menor número de escamas correspondió a 26 esc./cm proveniente de dos individuos y el mayor a $38 \mathrm{esc} . / \mathrm{cm}$.

Figura 2. Variación en la forma de la verruga sobre el surco nasolabial del rinario de $C$. aroensis en el Parque Nacional Yurubí, Venezuela. A .- pequeña y restringida a la base del rinario (MZUC-1012); B.larga, pero no alcanzando la boca (MZUC-1013); C.larga y delgada, extendida desde la base del rinario hasta la boca (EBRG29385).

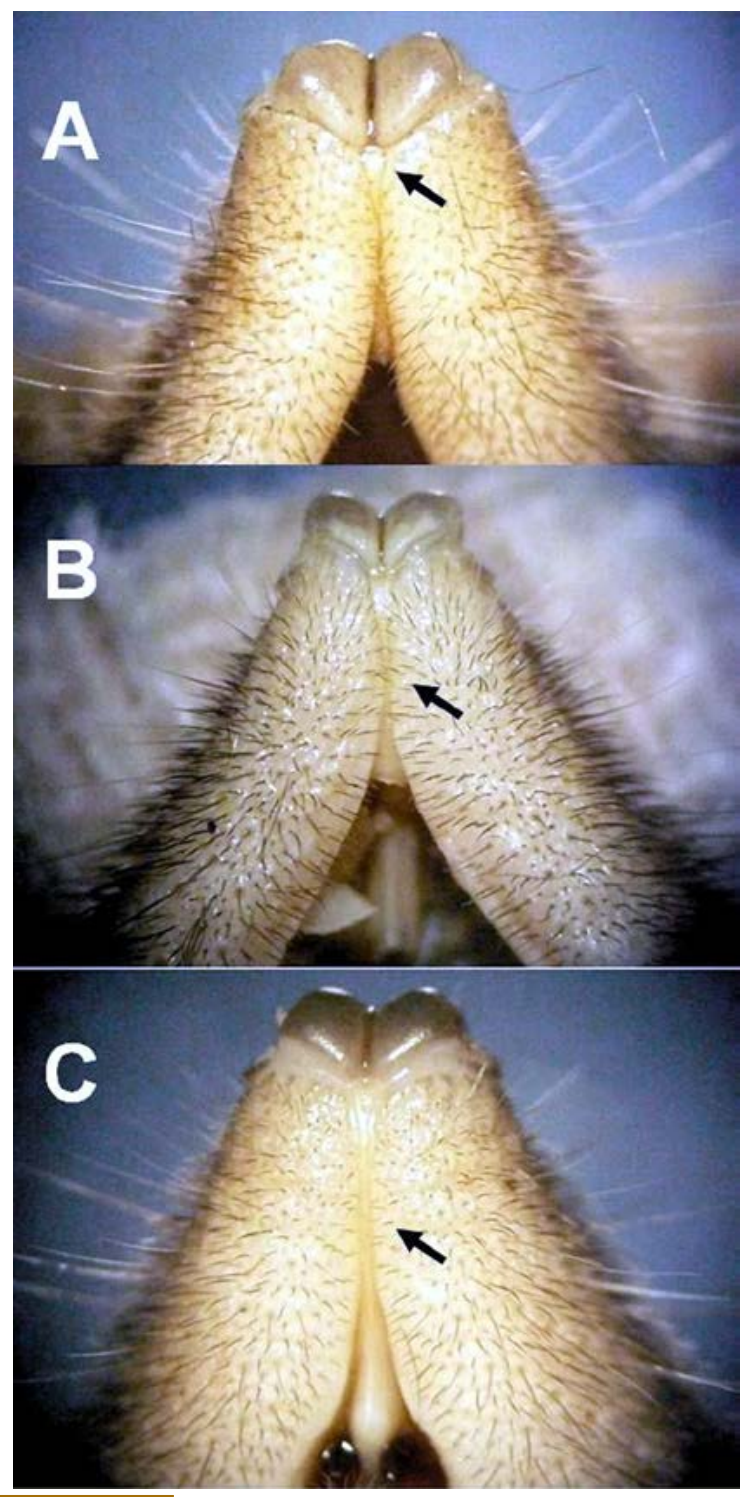

La pigmentación de la fila de dientes superiores e inferiores en individuos con los cráneos extraídos disponibles $(n=19)$ varió de la siguiente manera: marcada en todos los dientes superiores (excepto el cuarto unicúspide) y en todos los dientes inferiores $(n=$ 19); o marcada en casi todos los dientes superiores (a excepción del cuarto unicúspide, que no posee coloración; el tercer unicúspide y tercer molar superior son más pálidos) y marcada en todos los dientes inferiores (EBRG-29384); o marcada en todos los dientes superiores (excepto el cuarto unicúspide, que no tiene coloración y tercer unicúspide, cuya coloración es muy pálida) y en la mandíbula marcada sólo en los incisivos y pálida en el resto (MZUC-1022). 
La presencia y visibilidad del cuarto diente unicúspide, varió de la siguiente manera: presente en ambos lados, reducido en comparación al tamaño de los unicúspides uno y tres (U1-U3) y visible en ambos lados del cráneo en vista labial (EBRG-29385, 29388, 29398; MZUC-1019, 1024, 1032, 1036, 1037, 1038); presente en ambos lados del cráneo, reducido en comparación al tamaño de los U1-U3 y no visible en ambas vistas (labial y lingual; EBRG-29383, 29384, 29386; MZUC-1033); presente en ambos lados del cráneo, reducido en comparación al tamaño de los U1-U3 y observado sólo del lado labial en uno de los laterales del cráneo (EBRG-29387; MZUC-1012, 1016, 1017, $1020,1021,1022)$. Un individuo (MZUC-1033) tiene el elemento posterior del ectolofo del primer molar superior del mismo tamaño que el posterior; para el resto, el elemento anterior es mayor al posterior.

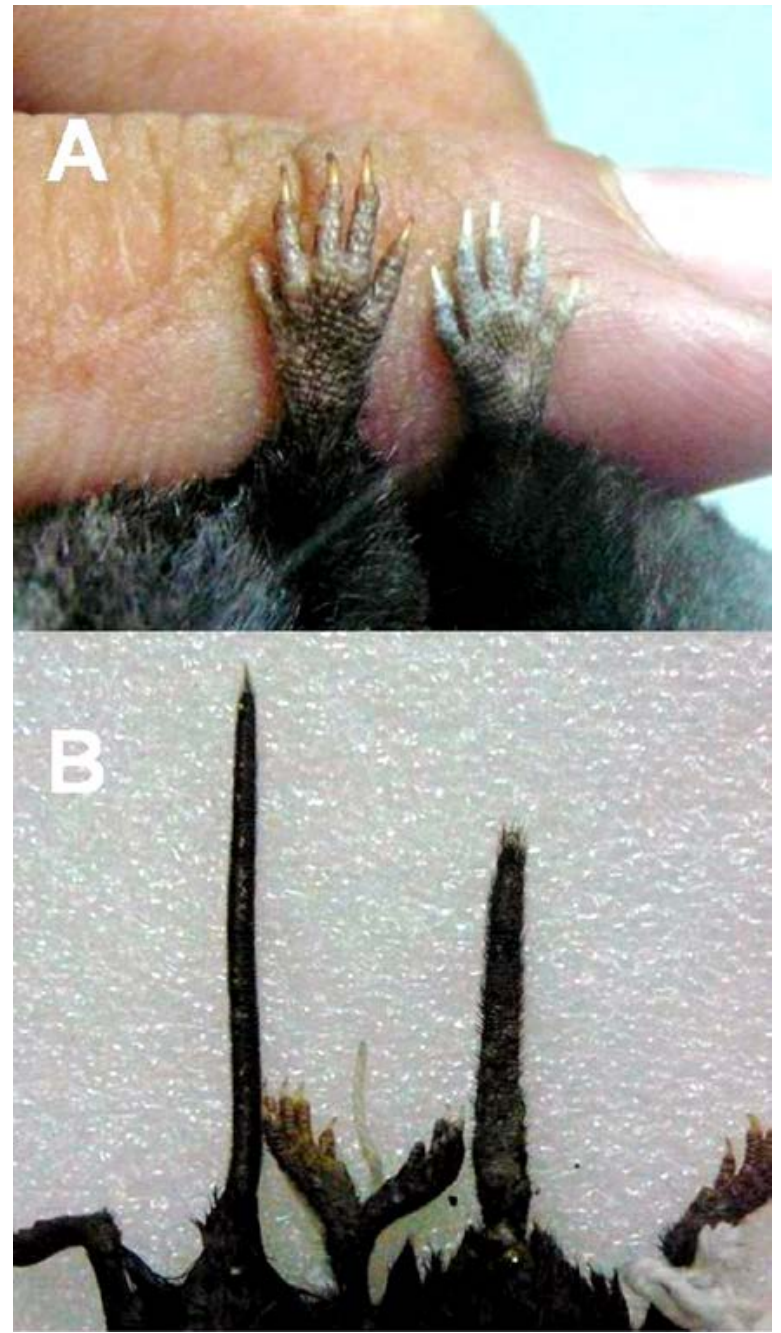

Figura 3. A.- Tamaños de las manos de $C$. meridensis (izquierda; MZUC-855) y C. aroensis (derecha; EBRG-29383). Se detalla el menor tamaño de los ejemplares de la serie del Parque Nacional Yurubí con respecto a C. meridensis. B.- Se observa una cola más larga en C. aroensis (izquierda; EBRG-29383) respecto a $C$. meridensis (derecha; MZUC-855) y al resto de las especies en Venezuela.

Los forámenes dorsales sobre el cráneo variaron de la siguiente manera: con dos forámenes dorsales grandes, de igual tamaño, y ubicados en la sutura entre los huesos frontales (EBRG-29383; MZUC-1020, 1034, 1035); dos forámenes de diferentes tamaños, ubicados sobre lados opuesto de la sutura de los huesos frontales (EBRG-29384, 29385, 29386, 29387; MZUC-1019, 1021, 1022, 1037); un foramen dorsal grande, sobre un lado de la sutura (EBRG-29398; MZUC-1012, 1017, 1032, 1038); sin presencia de algún foramen dorsal a lo largo de la sutura entre los frontales (MZUC-1036). 
La presencia o ausencia de un foramen sobre el borde posterior del proceso timpánico del petromastoideo varió de la siguiente manera: foramen ausente (EBRG-29383, 29384, 29398; MZUC-1016, 1020, 1022, 1033, 1034, 1036); o con un foramen en ambos procesos timpánicos (EBRG-29387; MZUC-1012, 1017, 1019, 1021, 1037); o un foramen sobre el lado derecho (EBRG-29386); o un foramen diminuto (casi imperceptible) sobre el lado izquierdo (EBRG-29385; MZUC-1024, 1035).

Figura 4. Tamaño, aspecto y forma de los húmeros izquierdos de dos especies de musarañas deVenezuela: A.- C. meridensis (MZUC861), B y C.- C. aroensis (EBRG-29398; MZUC1038). Escala $=4 \mathrm{~mm}$.

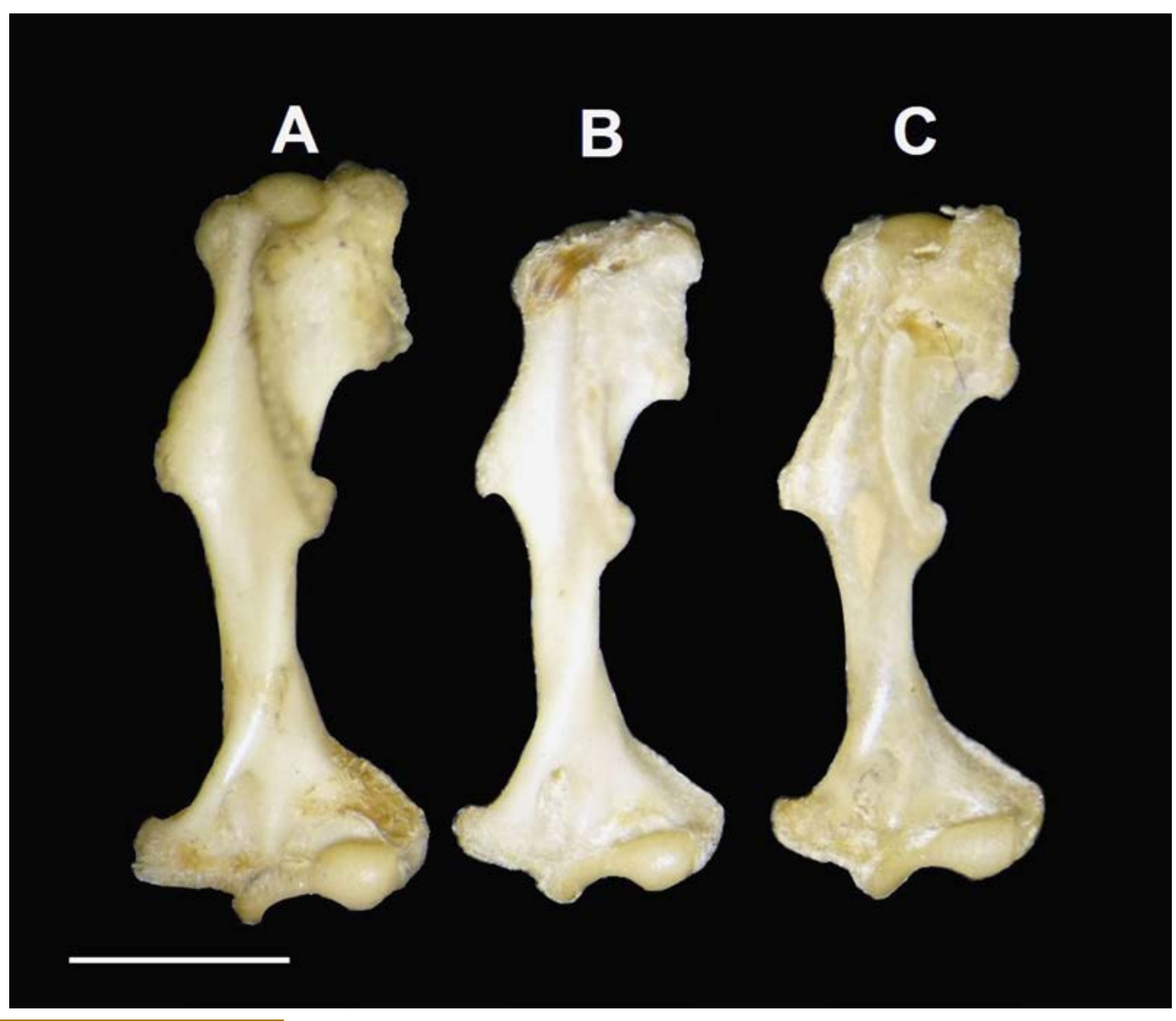

Las medidas promedio para dos húmeros disponibles (EBRG-29398; MZUC-1038; Fig. 4) fueron: longitud del húmero desde la cabeza hasta la tróclea $=14.26 \mathrm{~mm}$; distancia desde el tubérculo mayor hasta la tuberosidad deltoídea $=7.64 \mathrm{~mm}$; ancho del final proximal del húmero $=3.67 \mathrm{~mm}$; distancia interna desde el epicóndilo medio a la tuberosidad deltoídea $=5.84 \mathrm{~mm}$; ancho del eje desde la base superior del epicóndilo medio a la base superior del epicóndilo lateral $=1.90 \mathrm{~mm}$; distancia desde el proceso deltoide a la cabeza $=10.12 \mathrm{~mm}$ y ancho del final distal del húmero $=5.65 \mathrm{~mm}$.

\section{Discusión}

Los resultados de este trabajo coinciden con reportes previos que indican una elevada variación morfológica, cualitativa y cuantitativa, entre individuos de los grupos de Cryptotis nigrescens (Woodman y Timm 1993), C. goldmani (Woodman y Timm 1999, Woodman 2011), C. thomasi (Woodman 2002), C. mexicanus (Ramírez-Pulido et al. 2004) y C. parvus (Ramírez-Pulido et al. 2004). 
Por ejemplo, si se compara el alto rango de variación registrado en la población de C. aroensis del Parque Nacional Yurubí con un estudio de revisión taxonómica para $C$. meridensis en los Andes venezolanos (Woodman 2002), encontramos que para ambos trabajos se reporta una amplia variación morfológica intraespecífica, cuando se analizan series grandes y representativas. Por otra parte, para C. venezuelensis y C. tamensis no se reportan variantes morfológicas (Woodman 2002; Quiroga-Carmona 2013), presumiblemente por la baja representación en colecciones zoológicas venezolanas.

En cuanto a la forma y aspecto del húmero, que es un carácter taxonómico utilizado para asignar a las especies de Cryptotis a algunos de los grupos propuestos (Woodman et al. 2003) y no había sido descrito previamente para las formas venezolanas no andinas (Quiroga-Carmona y Molinari 2012; Quiroga-Carmona 2013), se concluye sin duda alguna que $C$. aroensis es un representante del grupo $C$. thomasi; aunque el húmero es un poco más pequeño, menos robusto y menos curvo comparado con otra especie del grupo (C. meridensis), para la cual se tiene un húmero disponible en colecciones venezolanas. El húmero en $C$. aroensis tiene una cabeza redonda, con el tubérculo mayor desarrollado. El tubérculo menor es ligeramente curvo. El epincódilo medio es robusto y de forma redonda. El epicóndilo lateral es menos desarrollado que el epicóndilo medio y más curvo. La tróclea es menos desarrollada que el capítulo. La tuberosidad deltoídea es desarrollada y está ubicada hacia la parte central del hueso. El proceso pectoral y deltoíde son desarrollados y robustos.

Con respecto a los caracteres documentados en la descripción original de C. aroensis (Quiroga-Carmona y Molinari 2012), hay similitudes cuando la muestra aumenta de tres (holotipo y dos paratipos) a 45 especímenes. Esos caracteres incluyen la longitud-cabeza cuerpo y la longitud de la mandíbula (Tabla 1), además de la presencia de cúspides sobre el tercer molar superior que definen una conformación de molar complejo. QuirogaCarmona y Molinari (2012), señalan que el paladar estrecho a nivel de los segundos molares superiores es el único rasgo distintivo que separa a $C$. aroensis del resto de las especies del grupo C. thomasi. Con esta nueva serie del Parque Nacional Yurubí, este atributo sigue siendo un carácter que distingue a $C$. aroensis del resto de las especies de Venezuela (Tabla 1); sin embargo, aunque en promedio el paladar a nivel de los segundos molares es ligeramente más angosto, hay 18 individuos de la serie examinada de $C$. aroensis cuyas medidas para este carácter están dentro del intervalo de variación de las tres especies restantes. Adicionalmente, los resultados de esta revisión sugieren que la longitud de la cola en $C$. aroensis (extremadamente larga) puede ser un atributo morfométrico útil para separarlo de los diferentes taxones presentes en Venezuela y no tomado en cuenta en la descripción original como un carácter diagnóstico (QuirogaCarmona y Molinari 2012), presumiblemente por el pequeño número de ejemplares al momento de la descripción.

Cuando se compara la serie tipo de C. venezuelensis, una especie recientemente descrita sobre el basamento de tres ejemplares y que habita en la biorregión conocida como Cordillera de la Costa, al igual que C. aroensis (Quiroga-Carmona y Molinari 2012; Quiroga-Carmona 2013) se registran varias similitudes entre ambas especies en medidas externas y peso, además de caracteres morfológicos como la presencia y visibilidad del cuarto diente unicúspide, la forma de la verruga y extensión en el surco nasolabial del rinario, el número de verrugas post-nasales, el tamaño y forma de los 
procesos angulares y coronoides, el número de escamas de la cola en un centímetro y la coloración del cuerpo. Esta situación sugiere que estos taxones probablemente podrían estar cercanamente emparentados. En cualquier caso, son necesarios nuevos ejemplares, especialmente de $C$. venezuelensis, para efectuar una comparación taxonómica más detallada y documentar las variaciones morfológicas como las señaladas ahora para $C$. aroensis; además es muy importante realizar estudios ecológicos que ayuden a establecer los límites de alturas, requerimientos de hábitats, dieta entre otros aspectos, para ambas especies en la Cordillera de la Costa.

\section{Agradecimientos}

A H. M. Silva y A. Pérez Sarmiento por el traslado a los sitios de muestreo. A I. Díaz y V. Colmenares (Guache), por el hospedaje en los sitios de trabajos de campo. Al Departamento de Biología de la FACyT, Universidad de Carabobo, por el apoyo logístico. A E. Sánchez-González por la realización del mapa. Al Ministerio del Poder Popular para el Ambiente (MinAmb) y al Instituto Nacional de Parques (INPARQUES) por los permisos concedidos en su momento y finalmente a Sergio Solari y dos revisores anónimos cuyas sugerencias y correcciones ayudaron a mejorar la primera versión del manuscrito.

\section{Literatura citada}

García, F. J., M. I. Delgado-Jaramillo, M. Machado y L. Aular. 2013. Pequeños mamíferos no voladores de un bosque nublado del Parque Nacional Yurubí, Venezuela: abundancia relativa y estructura poblacional. Interciencia 38:719-725.

Quiroga-Carmona, M., y J. Molinari. 2012. Description of a new shrew of the genus Cryptotis (Mammalia: Soricomorpha: Soricidae) from the Sierra de Aroa, an isolated mountain range in northwestern Venezuela, with remarks on biogeography and conservation. Zootaxa 3441:1-20.

Quiroga-Carmona, M. 2013. Una nueva especie de musaraña del género Cryptotis (Soricomorpha: Soricidae) de la Serranía del Litoral en el norte de Venezuela. Mastozoología Neotropical 20:123-137.

Ramírez-Pulido, J., A. Castillo-Morales, A. Salame-Méndez, y A. Castro-Campillo. 2004. Características morfológicas y morfométricas de cinco especies de Cryptotis (Mammalia: Soricomorpha). Acta Zoológica Mexicana 20:9-37.

Sánchez-HeRnández, J., y D. Lew. 2012. Lista actualizada y comentada de los mamíferos de Venezuela. Memoria de la Fundación La Salle de Ciencias Naturales 173174:173-238.

Woodman, N. 2002. A new species of small-eared shrew from Colombia and Venezuela (Mammalia: Soricomorpha: Soricidae: Genus Cryptotis). Proceedings of the Biological Society of Washington 115:249-272.

Woodman, N. 2003. A new small-eared shrew of the Cryptotis nigrescens-group from Colombia (Mammalia: Soricomorpha: Soricidae). Proceedings of the Biological Society of Washington 116:853-872.

Woodman, N. 2011. Patterns of morphological variation amongst semifossorial shrews in the highlands of Guatemala, with the description of a new species (Mammalia, Soricomorpha, Soricidae). Zoological Journal of the Linnean Society 163:12671288. 
Woodman, N., y R. M. Tıмm. 1993. Intraspecific and interspecific variation in the Cryptotis nigrescens species complex of small-eared shrews (Insectivora: Soricidae), with the description of a new species from Colombia. Fieldiana Zoology 1452:1-30.

Woodman, N., Y R. M. Tıмm. 1999. Geographic variation and evolutionary relationships among broad-clawed shrew of the Cryptotis goldmani-group (Mammalia: Insectivora: Soricidae). Fieldiana Zoology 91:1-35.

Wooman, N., C. A. Cuartas-Calle, y C. A. Delgado-V. 2003. The humerus of Cryptotis colombiana and its bearing on the species' phylogenetic relationships (Soricomorpha: Soricidae). Journal of Mammalogy 84:832-839.

Sometido: 29 de marzo de 2014

Revisado: 15 de junio de 2014

Aceptado: 26 de junio de 2014

Editor asociado: Sergio Solari

Diseño gráfico editorial: Gerardo Hernández

Especímenes examinados:

Cryptotis aroensis: La Trampa del Tigre $\left(10^{\circ} 24^{\prime} 11^{\prime \prime} \mathrm{N}-68^{\circ} 48^{\prime}\right.$ 01" O; 1,400-1,940 m), Sector El Silencio Parque Nacional Yurubí, Sierra de Aroa, Yaracuy (EBRG-29383 al 29389; MZUC-1010 al 1038).

Cryptotis meridensis: Mérida, Monte Zerpa, 6 Km N, Mérida, 2,162 m (MZUC-853 al 862). 